Check for updates

Cite this: Phys. Chem. Chem. Phys., 2017, 19, 11510

Received 2nd February 2017. Accepted 23rd March 2017

DOI: $10.1039 / c 7 c p 00728 k$

rsc.li/pccp

\title{
Electrolytic conductivity-related radiofrequency heating of aqueous suspensions of nanoparticles for biomedicine $\dagger$
}

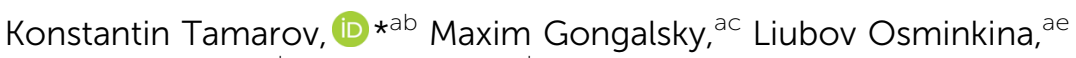 \\ Yuanhui Huang, ${ }^{\text {cd }}$ Murad Omar, ${ }^{\text {cd }}$ Valery Yakunin, ${ }^{a}$ Vasilis Ntziachristos, ${ }^{\text {cd }}$ \\ Daniel Razansky (iD ${ }^{c d}$ and Victor Timoshenko ${ }^{\text {ae }}$
}

\begin{abstract}
The development of suitable contrast agents can significantly enhance the efficiency of modern imaging and treatment techniques, such as thermoacoustic (TA) tomography and radio-frequency (RF) hyperthermia of cancer. Here, we examine the heating of aqueous suspensions of silicon (Si) and gold ( $\mathrm{Au}$ ) nanoparticles (NPs) under RF irradiation in the MHz frequency range. The heating rate of aqueous suspensions of Si NPs exhibited non-monotonic dependency on the electrical conductivity of the suspension. The experimental results were explained by the mathematical model considering oscillating solvated ions as the main source of Joule heating. These ions could be the product of the dissolution of Si NPs or organic coating of Au NPs. Thus, the ions governed the conductivity of the suspensions, which in turn governs both the heating rate and the near-field RF TA response. The model predicted the contrast in different tissues taking into account both Joule heating and dielectric losses.
\end{abstract}

\section{Introduction}

The ability of radio frequency (RF) radiation to heat human tissues has been known for a long time, but after recent developments of thermoacoustic (TA) tomography and demonstration of local destruction of tumors by invasive RF ablation, RF hyperthermia is attracting renewed interest. ${ }^{1,2}$ These techniques rely on the local hyperthermia - a procedure of raising the temperature of tissues using Joule heating. For example, in TA imaging, the contrast is produced by the differential absorption of microwave (MW) or RF radiation by tissues. ${ }^{3,4}$ However, because of the similar electromagnetic properties of tissues, achieving a good contrast is challenging but can be enhanced by new contrast agents (CAs).

\footnotetext{
${ }^{a}$ Faculty of Physics, M. V. Lomonosov Moscow State University, 119991 Moscow, Russia. E-mail: konstantin.tamarov@uef.fi

${ }^{b}$ Department of Applied Physics, University of Eastern Finland, 70211 Kuopio, Finland

${ }^{c}$ Institute of Biological and Medical Imaging, Helmholtz Zentrum München, 85764 Neuherberg, Germany

${ }^{d}$ Chair of Biological Imaging, Technische Universität München, 80333 Munich, Germany

${ }^{e}$ National Research Nuclear University "MEPhI", International Laboratory "Bionanophotonics", 115409 Moscow, Russia

$\dagger$ Electronic supplementary information (ESI) available: Includes the spectrum of TA emission, structural characterization of Si NPs, heating rates of $\mathrm{NaCl}$ solutions at $27.12 \mathrm{MHz}$, heating rates of electrolytes and suspensions of Si NPs at $1.2 \mathrm{MHz}$ and $40.68 \mathrm{MHz}$, and heating rates of aqueous suspensions of Si NPs before and after centrifugation. See DOI: 10.1039/c7cp00728k
}

In the case of RF ablation, the heating is performed by introducing needle-like electrodes directly into the tumor, ${ }^{5}$ which affect both tumor and normal tissues. Therefore dealing with big tumors can be problematic and requires additional cooling of the needles or constant infusion of saline for the enhancement of heating. ${ }^{6}$ Therefore, the development of RF-absorbing sensitizers is of importance in devising minimally invasive and cost-effective techniques for the thermal treatment of cancer. Due to the similar heating mechanism, such CAs can also be beneficial for diagnostic modalities based on RF/MW absorption, such as near-field RF TA imaging. ${ }^{4,7}$ Taken together, the RF-absorbing agents open the possibilities for simultaneous diagnostic and treatment modalities targeted at cancerous diseases (so-called theranostics).

Gold nanoparticles (Au NPs) have attracted attention as possible RF-absorbing agents in the last decade, ${ }^{8-10}$ and they were successfully used for non-invasive treatment of cancer. ${ }^{11}$ However, the underlying mechanisms of RF heating of Au NPs remain controversial: some groups have reported the absence of heating for uncoated $\mathrm{Au}$ NPs in deionized water. ${ }^{12,13}$ A number of explanations were proposed to describe the Au NP hyperthermia effect, including Joule heating, ${ }^{14}$ classical Mie's theory, ${ }^{15}$ quantum mechanisms, ${ }^{15}$ electrophoresis ${ }^{16}$ and polarization. ${ }^{17}$ Also, Si-based NPs have been observed to provide strong temperature increases of suspensions and then suppression of tumor growth in mice models under RF irradiation at $27.12 \mathrm{MHz}{ }^{18}$ There, the heating effect was qualitatively explained by taking into account the ionic currents on the surface of NPs. 
In the present work, we investigated the mechanisms responsible for the heating of aqueous suspensions of solidstate NPs, i.e. porous silicon (PSi) NPs, ablated Si (ASi) NPs, Si nanowires (NWs), and Au NPs as a result of their interaction with RF fields of various frequencies. Furthermore, Si NPs and NWs were assessed as possible TA contrast agents. Finally, we proposed a model considering collective oscillations of solvated ions (e.g. protons) in a viscous liquid medium as a key mechanism of heating. In order to verify the model, we additionally carried out experiments with electrolyte solutions of different $\mathrm{pH}$ values and conductivities. Considering the conductivity of the electrolytes allowed the calculation of the contrast ratio between the medium containing NPs and the reference medium, i.e. deionized water and biological tissues. The obtained results are discussed from the viewpoint of application as RF ablation sensitizers and TA contrast agents.

\section{Experimental procedure}

Electrolyte solutions with different conductivities were prepared by adding necessary amounts of $\mathrm{HCl}$ or $\mathrm{NaCl}$ into deionized water $(18.2 \mathrm{M} \Omega \mathrm{cm})$. PSi NP fabrication details can be found elsewhere. ${ }^{18-20}$ Briefly, PSi films were formed by electrochemical etching (anodization) of boron-doped crystalline $\mathrm{Si}$ (c-Si) wafers of specific resistivity $\rho=1-10 \mathrm{M} \Omega \mathrm{cm}$ and $10 \Omega \mathrm{cm}$ to obtain mesoporous Si (MPSi) and microporous Si ( $\mu \mathrm{PSi}$ ) layers, respectively. The anodization was performed in $\mathrm{HF}(48 \%): \mathrm{C}_{2} \mathrm{H}_{5} \mathrm{OH}$ solution (1:1) at a current density of $60 \mathrm{~mA} \mathrm{~cm}^{-2}$ and an etching time of $60 \mathrm{~min}$. The porosity of the as-prepared samples was $70 \pm 5 \%$, as determined by weight measurements. ${ }^{21}$ The PSi films were then ball-milled in deionized water in a planetary mill (Pulverisette, 7 premium line, Fritsch, Germany) for $30 \mathrm{~min}$ with $2.5 \mathrm{~mm}$ zirconium oxide balls and $30 \mathrm{~min}$ with $0.1 \mathrm{~mm}$ ones. After milling, centrifugation (Eppendorf Centrifuge 5424, Eppendorf, Germany) for $10 \mathrm{~min}$ at $1000 \mathrm{~g}$ was performed to obtain 50-200 $\mathrm{nm}$ sized particles.

Si NWs were fabricated by metal (silver)-assisted chemical etching (MACE) of Si wafers of specific resistivity $\rho=1-10 \mathrm{~m} \Omega \mathrm{cm}$ and $10 \Omega \mathrm{cm} .{ }^{22}$ Firstly, to form silver NPs on the surface of the Si wafers, they were immersed into a $0.03 \mathrm{M}$ aqueous solution of silver nitrate $\left(\mathrm{AgNO}_{3}\right)$ and $5 \mathrm{M}$ of $\mathrm{HF}$ in a volume ratio of $1: 1$. Secondly, Ag-coated Si wafers were placed into the solution of $5 \mathrm{M} \mathrm{HF}$ and $30 \% \mathrm{H}_{2} \mathrm{O}_{2}$ in the volume ratio 10:1 for 1 hour. Finally, the layer of Si NWs grown on the substrate was rinsed several times and dried in the air at room temperature. Si NWs were then detached from the substrate by sonication in an ultrasound bath (Elmasonic S10H, Elma Schmidbauer, Germany) in distilled water for 3 hours. Note that MACE of highly doped (HD) wafers $(\rho=1-10 \mathrm{~m} \Omega \mathrm{cm})$ led to the formation of porous $\mathrm{Si}$ NWs, whereas etching of lowly doped (LD) wafers $(\rho=1-10 \Omega \mathrm{cm})$ resulted in non-porous Si NWs.

To prepare NPs by laser ablation, a Si or Au target was placed at the bottom of a glass vessel filled with $20 \mathrm{~mL}$ of deionized water. Radiation from a Yb:KGW femtosecond laser (Amplitude Systems, Pessac, France, 1025 nm, 480 fs, 500 mJ, 1-5 kHz) was focused with the help of a $750 \mathrm{~mm}$ lens onto the target surface to provide the ablation of the target. ${ }^{23-25}$ The target was moved at a scanning velocity of $0.35 \mathrm{~mm} \mathrm{~s}^{-1}$ in the focusing plane to obtain identical surface conditions during the laser ablation, while the thickness of the water layer above the target was about $1 \mathrm{~cm}$.

The RF heating experiments at $27.12 \mathrm{MHz}$ and $40.68 \mathrm{MHz}$ were performed by applying standard medical apparatus for physiotherapy with maximum powers of $66 \mathrm{~W}$ and $60 \mathrm{~W}$, respectively. Each apparatus consists of a power source and a pair of electrodes of diameter $40 \mathrm{~mm}$ with a distance of $40 \mathrm{~mm}$ between them. The heating systems for frequencies of $1.2 \mathrm{MHz}$ and 4.1 MHz consisted of a generator for the corresponding frequency, an amplifier, and two electrodes. For $1.2 \mathrm{MHz}$ the same electrodes as described previously were used, while for $4.1 \mathrm{MHz}$ two electrodes of diameter $78 \mathrm{~mm}$ with a separation of $35 \mathrm{~mm}$ were used. The estimated field strengths at 1.2 $\mathrm{MHz}, 4.1 \mathrm{MHz}, 27.12 \mathrm{MHz}$ and $40.68 \mathrm{MHz}$ were equal to $20 \mathrm{~V} \mathrm{~cm}^{-1}, 90 \mathrm{~V} \mathrm{~cm}^{-1}, 90 \mathrm{~V} \mathrm{~cm}^{-1}$ and $30 \mathrm{~V} \mathrm{~cm}^{-1}$, respectively, which were similar to the values measured using an antenna connected to an oscilloscope. The temperature of the aqueous suspensions of Si NPs and NWs and saline solutions was measured using an infra-red thermometer (A\&D DT-633, A\&D, Japan) after switching off the power sources. To investigate the heating process, $8 \mathrm{ml}$ glass cuvettes were filled with the solution and placed in the center, so there always was an air gap between the electrodes and the glass cuvette. The electrical conductivity of the solutions was measured by using an HP 4192A LF impedance analyzer.

A hand-made near-field RF TA (NRT) imaging setup consisted of a custom-made high-voltage impulse generator providing $50 \mathrm{~ns}$ (FWHM) impulses carrying $50 \mathrm{~mJ}$ of energy. The emission spectrum is presented in the ESI $\dagger$ (Fig. S1). The induced TA response was detected using a PZT ultrasound transducer with a central frequency of $3.5 \mathrm{MHz}$, which was attached to automatic translational stages for $2 \mathrm{D}$ scanning. The suspensions were put into a rigid low-density polyethylene tube with matched acoustic impedance. The content of the tube could be replaced by two syringes connected to its holes. The coil for inducing the thermoacoustic signals, the transducer and the tube were all submerged in deionized water.

\section{Model and calculation results}

Fig. 1 shows the temperature increase of aqueous suspensions of Si NPs under RF irradiation with a power of $66 \mathrm{~W}$ and a frequency of 27.12 MHz. NP suspensions were found to be acidic with different $\mathrm{pH}$ values for different types of Si NPs and the decrease in $\mathrm{pH}$ values corresponded to the increase of suspension conductivity (Fig. S2, ESI $\dagger$ ). Typically, the conductivities were higher for the samples containing Si NPs with higher porosity and bigger surface area (Fig. S3, ESI $\dagger$ ). The suspensions of non-porous LD Si NWs were less heated and had lower conductivity (5 $\mathrm{mS} \mathrm{m}^{-1}$ ) in comparison to the mesoporous (MPSi, $9.5 \mathrm{mS} \mathrm{m}^{-1}$ ), microporous ( $\mu$ PSi, $31 \mathrm{mS} \mathrm{m}^{-1}$ ) and highly 


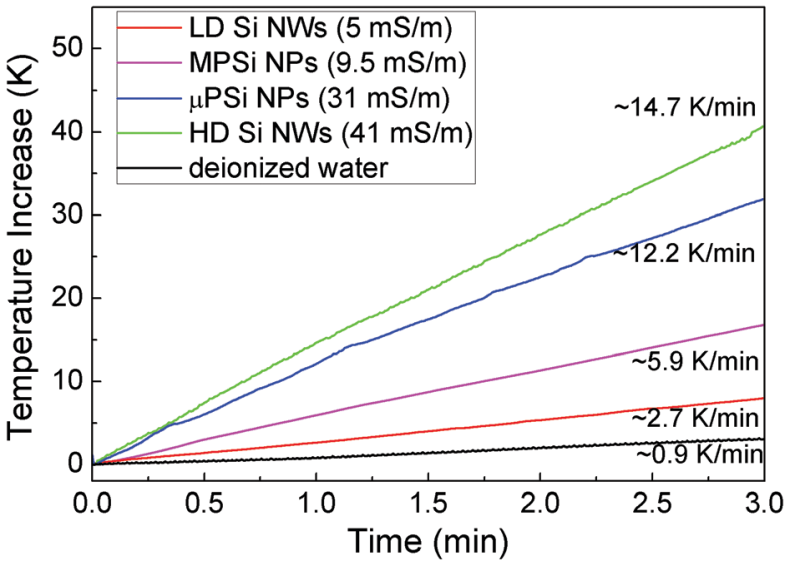

Fig. 1 Temperature increase of aqueous suspensions of Si NPs under RF irradiation $(66 \mathrm{~W})$ and frequency $27.12 \mathrm{MHz}$ versus the RF irradiation time. The concentrations are $1 \mathrm{mg} \mathrm{ml}^{-1}$ for MPSi NPs (magenta line) and $\mu$ PSi NPs (blue line), $250 \mu \mathrm{g} \mathrm{ml}^{-1}$ for LD Si NWs (red line) and HD Si NWs (green line). The conductivity values of the suspensions are specified in the legend.

porous HD Si NWs $\left(41 \mathrm{mS} \mathrm{m}^{-1}\right)$ samples. In order to clarify the conductivity effect on the heating, a set of electrolytes without NPs were prepared. The conductivity values of the electrolytes varied in the range from $5 \times 10^{-3} \mathrm{~S} \mathrm{~m}^{-1}$ to $10^{2} \mathrm{~S} \mathrm{~m}^{-1}$. The heating rates of suspensions of Si NPs, Au NPs and several electrolytes without NPs were measured under RF irradiation at 1.2 MHz (Fig. S4, ESI $\dagger$ ), 4.1 MHz (Fig. 2a), 27.12 MHz (Fig. 2b) and $40.68 \mathrm{MHz}$ (Fig. S5, ESI $\dagger$ ). The experimental dependency had a distinguished maximum at a certain conductivity level for all frequency values. As the frequency increased, the maximum moved to higher conductivities and the heating became more efficient. The observed experimental results were explained by a model, which takes into account only the electrical conductivity of the investigated suspensions and it successfully described the heating effect (red line in Fig. 2).

Similar results were obtained by the near-field RF TA tomography (NRT) experiments. The set of aqueous suspensions was irradiated by RF pulses in the window of $3-12 \mathrm{MHz}$ and thermally induced acoustic waves were detected. Basically, the method provided 2D or 3D-images of an object, governed by its TA response. A typical image of a straw filled with a suspension of NPs is shown in the inset of Fig. 3. Such images were used to obtain average integrated TA responses for each sample. Fig. 3 shows the conductivity dependency of Si NP suspensions and saline solutions. All the points lie perfectly on the red line, which can be considered as the calibration curve. This led to non-linear, but monotonic dependency of the TA response on the sample conductivity. The RF irradiation spectrum and horizontal TA profiles of the tubes are presented in the ESI $\dagger$ (Fig. S1).

Nevertheless, conductivity remained the major factor influencing TA intensity and, consequently, the efficiency of heating. This also confirmed the idea that both RF heating and TA waves were governed mostly by ionic oscillations in solutions rather than electrons in solid NPs. The latter are not supposed to

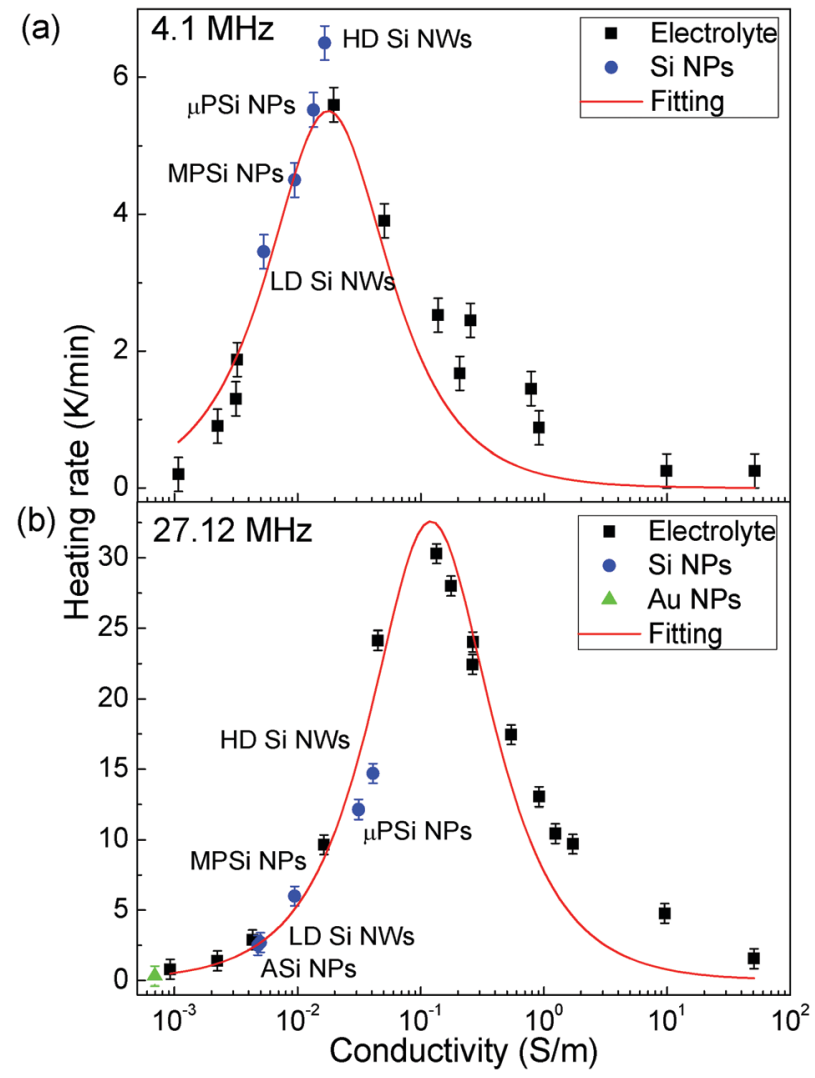

Fig. 2 (a) The dependency of heating rates $(S(\sigma))$ on conductivity of the electrolyte (black squares), conductivity of the aqueous suspension of $\mathrm{Si}$ (blue circles) and Au (green triangles) NPs, and fitting of the electrolyte heating rates according to the model (eqn (8), red line) at $4.1 \mathrm{MHz}$. (b) The same at $27.12 \mathrm{MHz}$.

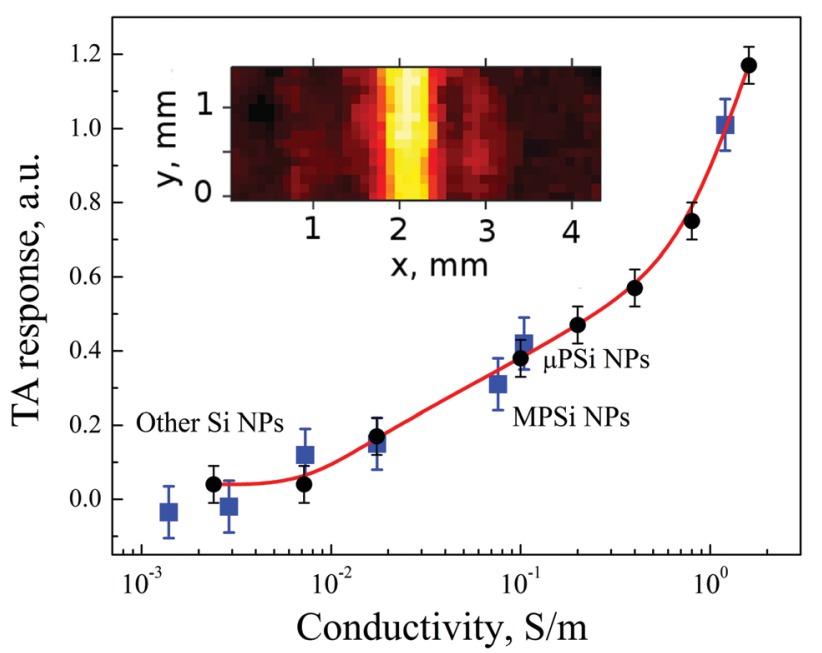

Fig. 3 Thermoacoustic response of NP suspensions (blue squares) and saline solutions (black circles) versus their electrolytic conductivity. The red curve shows the interpolation.

contribute to RF heating, because their plasmonic frequency is too high, therefore even low-conducting NPs perfectly reflect electromagnetic waves instead of absorbing them. 


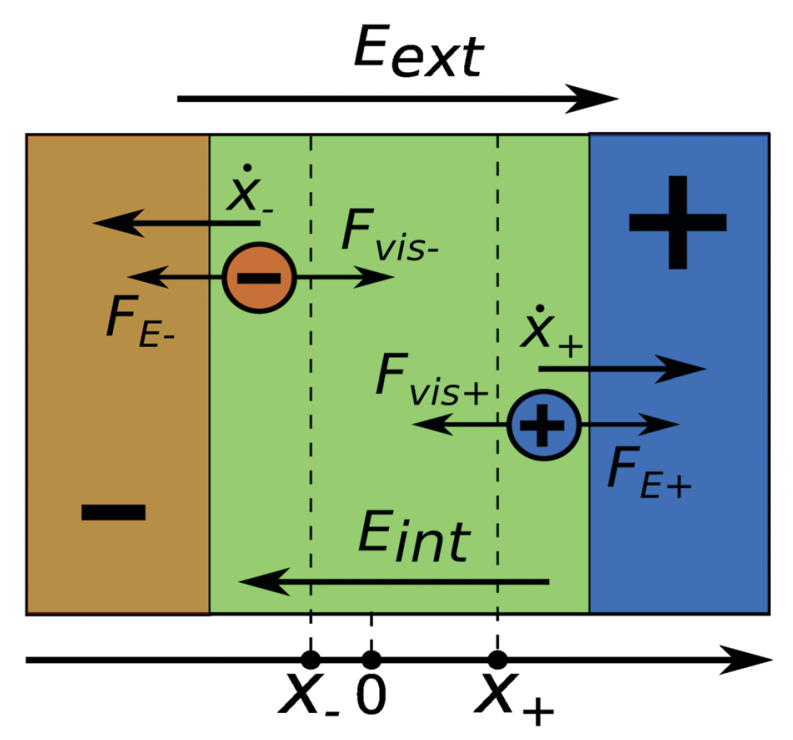

Fig. 4 A schematic view of the electrolyte affected by an external RF electromagnetic field $\left(E_{\text {ext }}\right)$. The positively and negatively charged regions are represented by blue and brown areas, respectively. Coordinates $x_{+}$and $x_{-}$show the displacement of positively and negatively charged regions. The displacement of the regions generates the internal electromagnetic field $\left(E_{\text {int }}\right)$ in the electrolyte.

In the experimental results, it was supposed that the solvated ions were the primary source of temperature increase in the electrolytes exposed to the radiation at RF frequencies below $1 \mathrm{GHz}$. The oscillating ions converted the energy of the electromagnetic field into heat via viscous friction with surrounding liquid (Fig. 4). The behavior of a single ion can be described by Newton's second law of motion:

$$
m \ddot{x}=F_{\mathrm{E}}-F_{\mathrm{vis}},
$$

where $m$ and $\ddot{x}$ are the mass and acceleration of the ion, respectively, $F_{\mathrm{E}}$ and $F_{\mathrm{vis}}$ are the total electromagnetic and viscosity friction forces applied to the ion, respectively. Here, $F_{\mathrm{E}}$ takes into account both the external RF radiation and polarization of liquid induced by interactions with all other ions in the liquid. The following assumptions were used to derive the equations of motion:

- Positive and negative ions are uniformly arranged in the volume, have charges $+q$ and $-q$, respectively, and are represented as two areas equal in magnitude and opposite in charge density (blue and brown areas in Fig. 4). The electrolyte is neutral in the absence of external forces. For convenience of description, these uniformly charged areas are considered as undeformable regions, which can move relative to each other. When the external electromagnetic field is applied, the charged areas are displaced by an offset from their equilibrium position in the opposite directions, i.e. displacements of positively and negatively charged areas are $x_{+}$and $-x_{-}$, respectively.

- The movement of the areas instantaneously achieves a quasi-stationary regime because of a strong viscous friction, i.e. the acceleration is neglected and the averaged Brownian random force is equal to zero. In this case, one can use the concept of mobility, $\mu$, which can be expressed as: $\mu=\dot{x} / E_{\text {total }} \cdot \mu_{+}$and $\mu_{-}$values are used for positive and negative ions, respectively.

- The relative dielectric permittivity of the electrolytes, $\varepsilon$, equals the relative permittivity of water and does not depend on RF frequency in the range of 1-100 $\mathrm{MHz}$ and concentration of solvated ions. More careful calculations can be potentially performed by using the experimental data summarized in ref. 26.

- The skin-effect in the electrolyte is ignored, because its depth is estimated to be in the range of $0.6 \mathrm{~m}$ and $160 \mathrm{~m}$ in the investigated range of conductivities and $\mathrm{RF}$ frequencies. The estimation was done according to the equation for the skin depth: $\delta=\left(2 / \sigma \omega_{0} \mu_{0}\right)^{1 / 2}$, where $\sigma$ is the electrical conductivity of the electrolyte, $\omega_{0}$ is the frequency of RF field and $\mu_{0}=4 \pi \times 10^{-7} \mathrm{H} \mathrm{m}^{-1}$.

The total electric field strength, $E_{\text {total }}$, consists of the external RF field, $E_{\text {ext }}$ and the internal field of the electrolyte polarization, $E_{\text {int }}$. For calculation of $E_{\text {int }}$ one can use the formula for two oppositely charged planes $E_{\text {int }}=-\Sigma_{\mathrm{E}} / \varepsilon \varepsilon_{0}$, where $\Sigma_{\mathrm{E}}$ is the surface charge density, $\varepsilon$ is the relative permittivity of the electrolytes and $\varepsilon_{0}=8.85 \times 10^{-12} \mathrm{~F} \mathrm{~m}^{-1} . \Sigma_{\mathrm{E}}$ can be considered as $\Sigma_{\mathrm{E}}=q n\left(x_{+}-x_{-}\right)$ and the expression for $E_{\text {total }}$ will be:

$$
E_{\text {total }}=E_{\text {ext }}+E_{\text {int }}=E_{\text {ext }}-\frac{q n}{\varepsilon \varepsilon_{0}}\left(x_{+}-x_{-}\right)= \pm \frac{\dot{x}_{ \pm}}{\mu_{ \pm}},
$$

where $q$ is the absolute charge of an ion, $n$ is the concentration of ions, $E_{\text {ext }}(t)=E_{0} \sin \left(\omega_{0} t\right)$ is the electric field of RF radiation, $E_{0}$ is the amplitude of RF radiation and sign \pm corresponds to the positively or negatively charged areas.

Eqn (2) can be rewritten in the following way:

$$
\dot{x}_{ \pm} \pm \frac{q n \mu_{ \pm}}{\varepsilon \varepsilon_{0}}\left(x_{+}-x_{-}\right)= \pm E_{0} \mu_{ \pm} \sin \left(\omega_{0} t\right) .
$$

Eqn (2) shows, that $\dot{x}_{+} / \mu_{+}=-\dot{x}_{-} / \mu_{-}$, and subsequently $x_{+} / \mu_{+}=-x_{-} / \mu_{-}$. Therefore, relative displacement between both areas is the following:

$$
x_{+}-x_{-}=x_{+}\left(1+\frac{\mu_{-}}{\mu_{+}}\right)=-x_{-}\left(1+\frac{\mu_{+}}{\mu_{-}}\right) .
$$

Taking into account that the electrical conductivity related to both positive and negative ions $\sigma=q n \mu_{+}+q n \mu_{-}$and eqn (4), eqn (3) can be written as:

$$
\dot{x}_{ \pm}+\frac{\sigma}{\varepsilon \varepsilon_{0}} x_{ \pm}= \pm E_{0} \mu_{ \pm} \sin \left(\omega_{0} t\right)
$$

By solving eqn (5) the following expression can be obtained:

$$
x_{ \pm}(t)= \pm \frac{E_{0} \mu_{ \pm}}{\sqrt{\omega^{2}+\omega_{0}^{2}}} \cos \left(\omega_{0} t+\phi\right),
$$

where $\omega=\sigma / \varepsilon \varepsilon_{0}$, and $\phi=\arcsin \left(\omega / \sqrt{\omega^{2}+\omega_{0}^{2}}\right)$, where the reciprocal value of $\omega$ is proportional to the Maxwell relaxation time.

The heating rate produced by viscous friction of ions is obviously proportional to $P_{\mathrm{VI}}(t)=q n E_{\text {ext }}(t)\left(\dot{x}_{+}(t)-\dot{x}_{-}(t)\right)$. And the average absorbed power density will be:

$$
P_{\mathrm{VI}}(\omega)=\frac{1}{2} \cdot E_{0}^{2} \cdot \varepsilon \varepsilon_{0} \cdot \frac{\omega_{0}^{2} \omega}{\omega_{0}^{2}+\omega^{2}} .
$$


Table 1 Experimental and theoretical values of electrolyte conductivities $\sigma_{0}$, where the maximum heating occurs at various frequencies

\begin{tabular}{lll}
\hline Frequency $(\mathrm{MHz})$ & $\sigma_{0}$ from experiment $\left(\mathrm{S} \mathrm{m}^{-1}\right)$ & $\sigma_{0}$ from model $\left(\mathrm{S} \mathrm{m}^{-1}\right)$ \\
\hline 1.2 & $0.0053 \pm 0.0023$ & 0.0051 \\
4.1 & $0.0176 \pm 0.0021$ & 0.0173 \\
27.12 & $0.121 \pm 0.011$ & 0.115 \\
40.68 & $0.183 \pm 0.045$ & 0.173
\end{tabular}

Or in terms of conductivities:

$$
P_{\mathrm{VI}}(\sigma)=\frac{1}{2} \cdot E_{0}^{2} \cdot \frac{\sigma_{0}^{2} \sigma}{\sigma_{0}^{2}+\sigma^{2}}
$$

where $\sigma_{0}=\omega_{0} \varepsilon \varepsilon_{0}$.

Note, that despite there being no direct dependency of $P_{\mathrm{VI}}(\sigma)$ on viscosity, there is indirect dependency, because $\sigma=q n \mu$ and $\mu$ decreases with increase in viscosity.

The heating rate is calculated from the heat equation taking into account the negligible temperature exchange between the electrolyte and ambient air:

$$
S_{\mathrm{VI}}=\frac{1}{c \rho} \cdot P_{\mathrm{VI}} \cdot 60\left[\mathrm{~K} \mathrm{~min}^{-1}\right]
$$

where $c$ and $\rho$ are the specific heat capacity and density of water, respectively. The maximum heating rate is observed when $\sigma=\sigma_{0}$. The lower the frequency the smaller is the corresponding conductivity for maximum heating. Eqn (7) does not consider particular ion types and it was confirmed that the model is still applicable for $\mathrm{NaCl}$ solutions (Fig. S6, ESI $\dagger$ ). The model also allowed us to explain a similar experimental dependence of saline heating rate on its electrical conductivity at $13.56 \mathrm{MHz}$ obtained by Liu et al. ${ }^{12}$ However, in case of complex organic-inorganic mixtures, the change of dielectric permittivity leads to the shift of the peak value of conductivity, ${ }^{27}$ which should be taken into account for modeling of biological tissues. A similar shift was observed in solutions with kosmotropic or chaotropic compounds, because they can form or disrupt hydrogen bonds in aqueous medium. ${ }^{28}$

Table 1 demonstrates that the conductivities $\sigma_{0}$ corresponding to maximal heating obtained from approximation of the experimental data are in good agreement with the model. However, at 1.2 MHz the shape of the calculated peak differs from the experimental one due to slight deviation from the quasi-stationary regime.

The developed model shows a linear dependency of the maximum heating rate on frequency: $S_{\max }\left(\omega_{0}\right)=E_{0}{ }^{2} \times \varepsilon \varepsilon_{0} \times \omega_{0} / 4 c \rho$. Fitting of the experimental data allowed acquiring values $\omega_{\max }$ and heating rate $S_{\max }$. Fig. 5 shows the frequency dependence of maximum heating normalized by the corresponding field strength. The modeling and the experimental data seem to agree reasonably well with each other confirming the model in the $\mathrm{RF}$ region (1-40 $\mathrm{MHz}$ ).

\section{Discussion}

However, if one wants to model the heating process at ultrahigh frequencies, dielectric losses in the $\mathrm{GHz}$ frequency range

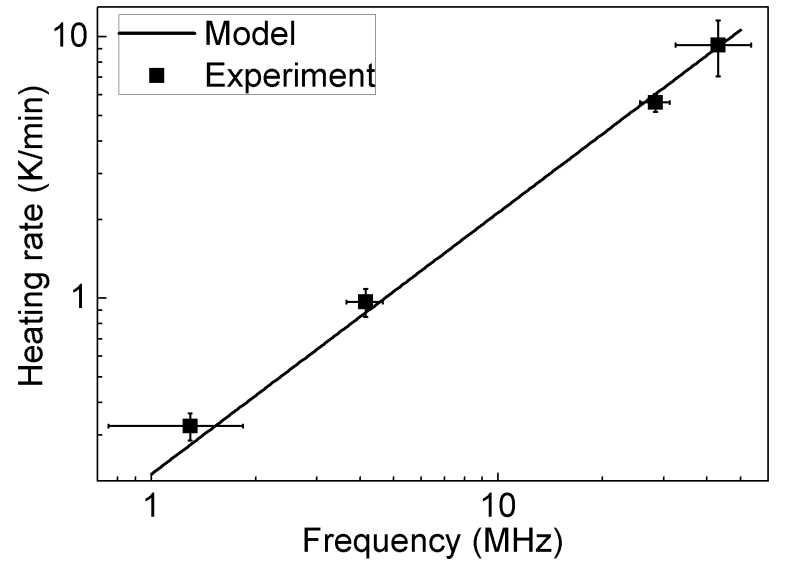

Fig. 5 Dependence of the maximum heating rate on the external electromagnetic field frequency, $S_{\max }\left(\omega_{0}\right)$.

must be taken into account. The power density released due to the dielectric losses in water can be described as follows:

$$
P_{\mathrm{DL}}=\frac{1}{2} \cdot \omega_{0} \varepsilon_{0} \varepsilon^{\prime \prime}\left(\omega_{0}\right) E_{0}^{2}
$$

where $\varepsilon^{\prime \prime}\left(\omega_{0}\right)$ is the imaginary part of the dielectric permittivity of water calculated according to the equations provided in ref. 29. Thus, the total heating rate of the solution can be represented as a sum of two items: heating rate induced by "viscous" ions $\left(S_{\mathrm{VI}}\right)$ and heating due to the dielectric losses $\left(S_{\mathrm{DL}}\right)$ :

$$
S=S_{\mathrm{VI}}+S_{\mathrm{DL}}=\frac{1}{c \rho} \cdot\left(P_{\mathrm{VI}}+P_{\mathrm{DL}}\right) \cdot 60\left[\mathrm{~K} \mathrm{~min}^{-1}\right]
$$

where $P_{\mathrm{VI}}$ and $P_{\mathrm{DL}}$ are described by eqn (7) or (8) and (10), respectively. In particular, $S_{\mathrm{VI}}$ depends on the conductivity of the solution and $S_{\mathrm{DL}}$ depends only on the dielectric losses of water. $S_{\mathrm{VI}}$ and $S_{\mathrm{DL}}$ are independent of each other and describe two physical mechanisms involved in the electromagnetic heating of water suspensions of Si NPs and modeling electrolytes.

It is known that the medical devices for physiotherapy are designed to produce the most efficient heating on biological tissues because the conductivities of different parts of the human body lie in the RF range of the corresponding frequencies. ${ }^{30}$ In order to find the most suitable therapeutic frequency, the contrast $\eta$ between the heating rates of different types of tissues and suspensions of Si NPs was calculated:

$$
\eta=\frac{S_{\mathrm{SiNPs}}-S_{\mathrm{tissue}}}{S_{\mathrm{SiNPs}}},
$$

where $S_{\text {SiNPs }}=S_{\text {VISiNPs }}+S_{\text {DLSiNPs }}$ is the maximum heating rate of the suspensions of Si NPs and $S_{\text {tissue }}=S_{\text {VItissue }}+S_{\text {DLtissue }}$ is the heating rate of the tissue. The values of $S_{\mathrm{VI}}$ and $S_{\mathrm{DL}}$ correspond to the calculation of heating rates according to eqn (7) and dielectric losses (eqn (10)) at the considered frequency. If the contrast value equals 0 , tissue and suspension have the same heating rates; so the contrast value of 1 is preferable.

Fig. 6 shows the simulated frequency dependence of the contrast for four types of tissues: muscle, bone, fat and blood; the conductivities of the tissues were taken from ref. 30. On the 


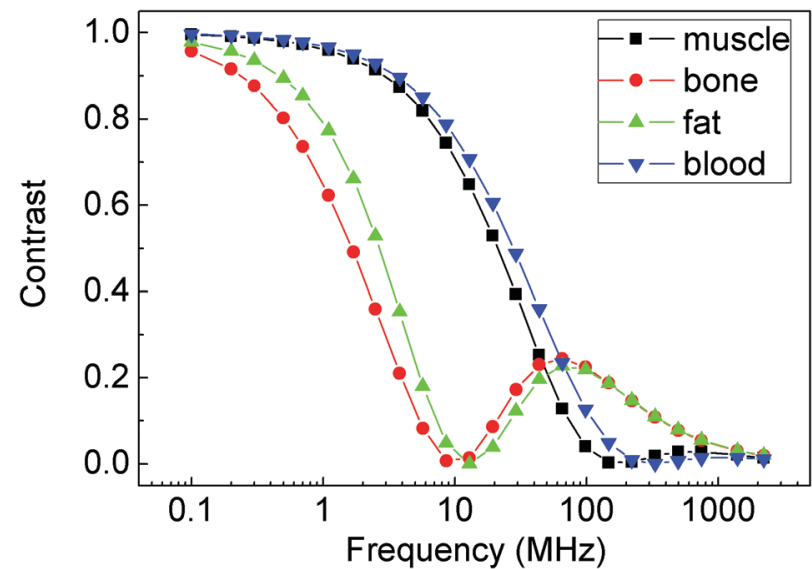

Fig. 6 Contrast values of the heating rate between a suspension of Si NPs and different tissues: muscle (black line), bone (red line), fat (green line) and blood (blue line) versus RF irradiation frequency, $\eta\left(\omega_{0}\right)$. The contrast was calculated by using eqn (12).

one hand, the contrast is quite low in the range of $2.5-60 \mathrm{MHz}$ for bone, fat and other tissues, which have conductivities in the 0.03-0.06 $\mathrm{S} \mathrm{m}^{-1}$ range. On the other hand, the contrast becomes low at frequencies higher than $70 \mathrm{MHz}$ for high conductivity tissues like muscle or blood. There is no measurable contrast between heating of DI water, suspensions of $\mathrm{Si}$ NPs and the electrolytes at $2.5 \mathrm{GHz}$ according to the measurements using MW heating equipment.

Therefore, it might be concluded that to obtain perfect contrast close to 1 and sufficient hyperthermia effect in the local area, the selected frequency should be about $1 \mathrm{MHz}$ or less. However, some difficulties arise at low frequencies. In particular, at lower frequencies, the conductivity value, where maximum heating is observed, is also low, which leads to a small temperature rise. Additionally, the shielding of the electromagnetic field by surrounding high conductivity tissues may occur, and it can reduce the heating rate of low conductivity materials. Consequently, the power of RF radiation must be increased in order to provide the same temperatures as at high frequencies. This gives rise to another problem, because the creation of such intense electromagnetic fields requires powerful sources and necessary protection to ensure the safety of patients. Thus, we suppose that the most convenient frequency is $70 \mathrm{MHz}$ as it gives the contrast of $25 \%$ between Si NPs and a tissue and this value does not depend on the type of biological tissue.

Some early publications attributed the temperature rise of $\mathrm{Au}$ NPs suspensions to the absorption of RF radiation by NPS themselves via Joule-like or inductive mechanisms. ${ }^{14,31}$ But although the high conductivity of gold can yield high heating rates when a current passes through it, in the external electromagnetic field free electrons in metals polarize easily, screen it, and prevent further heating. The negligible heating of Au NPs dispersed in deionized water was observed in our experiments and supports this assumption (Fig. S7, ESI $\dagger$ ).

In the second approach, the absorption cross-section of metal NPs suspensions is calculated according to Mie scattering theory. ${ }^{15,32}$ The authors reported that the negligible absorption of $\mathrm{RF}$ radiation by $\mathrm{Au}$ NPs is insufficient to heat the entire suspension, whereas the heating is more significant when the NPs are dispersed in a conductive medium; and this conclusion agrees with the developed model.

Thirdly, electrophoretic motion can be considered as a heat source because, typically, the commercially available Au NPs and Si NPs have significant net negative charge at neutral $\mathrm{pH}$ values. ${ }^{33,34}$ Under RF irradiation, charged particles can follow alternating electromagnetic fields. The imaginary component corresponding to power loss during electrophoresis was theoretically considered by Sassaroli ${ }^{32}$ and experimentally investigated by Corr. ${ }^{16}$ Although in the latter work Au NPs with diameters less than $10 \mathrm{~nm}$ had observable heat production at 13.56 $\mathrm{MHz}$, there is still no full understanding of the influence of the citrate coating. The electrophoresis seem to be more pronounced at lower frequencies, but the calculations suggest that this effect is not sufficient enough to explain the heating rates observed in our experiments.

Fourthly, the presence of a NP in the electrolyte can influence its polarization in the alternating $\mathrm{RF}$ field. ${ }^{17}$ There, the authors demonstrated that in the presence of the charged particle, the local conductivity near the particle is different from the conductivity of the bulk electrolyte due to the formation of an electrical double layer. ${ }^{35}$ However, although the particle did alter the heating, the high temperature rise was proposed at low conductivity values, which does not correspond to our data.

In the present work, it is argued if solid-state NPs are the main absorbers of RF radiation. Indeed, in the experiments, where significant heating was observed, citrate-coated Au NPs were used. ${ }^{8-11,14,16}$ On the contrary, several authors reported negligible heating rates for suspensions of non-functionalized $\mathrm{Au}$ NPs suspended in pure deionized water. ${ }^{12,13,36}$ Thus, the citrate coating dissolves and produces ions, which start to play a major role in the heating of suspensions. ${ }^{16}$ This conclusion is also supported by the work of Ogunlade and Beard, ${ }^{37}$ who tested gadolinium, iron oxide NPs and single-wall carbon nanotubes as potential TA CAs and demonstrated, that the NP coating was the main source of the contrast.

In order to exclude any influence of surface chemistry, which is usually fairly complicated for particles obtained by wet chemical approaches, ultrapure Au NPs were prepared by laser ablation of a gold target in deionized water. ${ }^{38}$ Hence, the suspension of Au NPs was obtained in low conductive medium, therefore the effect of intrinsic Au NPs heating would be evident, if it existing. However, the green point in Fig. 2 and the comparison with deionized water (Fig. S7, ESI $\dagger$ ) shows that the heating of the Au NP suspension can be completely described by the proposed model. Thus, the remarkable heating reported in the literature is governed mostly by contamination of the solvent by radicals which moved from surface coating to the solution. Similar results were observed for the TA response of $\mathrm{Au}$ NPs, which was also negligible.

In the case of Si NPs, they tend to dissolve in aqueous media forming considerable amounts of silicic acid. ${ }^{39}$ It leads to subsequent conductivity and heating rate increase of the surrounding liquid. To confirm the impact of the dissolution, 
the suspensions of Si NPs were centrifuged at $20000 \mathrm{~g}$ and the supernatant was filtered through a $0.2 \mu \mathrm{m}$ filter. The heating rates of the supernatant (Fig. S8, ESI $\dagger$ ) were similar to that of the suspensions shown in Fig. 1. When the sedimented NPs were resuspended in fresh deionized water, heating similar to water was observed (Fig. S9, ESI $\dagger$ ).

Despite the fact that the heating rate mostly depends on the ionic conductivity, Si NPs can be potentially used as a source of local conductivity increase and thus act as contrast agents for both therapy and diagnostics. The heating rate of Si NPs in low conductivity tissues could be much higher than the heating rate of the tissue itself, if appropriate frequency tuning is applied. Porous Si NPs can also be considered as compartments for liquid filling their pores. In this case, diffusion inside Si NPs is changed and intraporous liquid may maintain its conductivity for a long time. This may be also beneficial for the delivery of kosmotropes and triggered drug release techniques, because the surface of Si NPs can be coated by thermosensitive polymers, which change their conformation and open pore gates after reaching a certain temperature. ${ }^{40,41}$ Note, that the concentration dependence diffusion of liquids inside the pores ${ }^{42}$ should be taken into account for a correct description of RF heating of porous NPs.

Another promising application of solid-state NPs is NRT. ${ }^{4,7}$ Indeed, different materials have been tested for that purpose: graphene-based NPs, ${ }^{43,44}$ iron oxide NPs, ${ }^{45}$ and a Gd-based medical contrast agent. ${ }^{46}$ Graphene-based NPs demonstrated at best two-fold enhancement, while Gd NPs and iron oxide amplified the TA signal 3-fold and 6-fold, respectively, due to magnetic heating. However, in these studies MW radiation of 3 $\mathrm{GHz}$ was used, which penetrates around $0.7 \mathrm{~cm}$ into muscle tissue. ${ }^{45}$ Since Si NPs also demonstrated efficient TA response, they can be considered as a flexible framework for contrast enhancement in the $\mathrm{MHz}$ frequency region. The advantage of $\mathrm{Si}$ NPs over inorganic NPs mentioned above is their dissolution, which provides significantly better local heating around themselves. That in turn governs a stronger TA response of Si NPs. Moreover, the porous structure of Si NPs provides additional opportunities for contrast enhancement. TA contrast can be estimated, $\eta_{\mathrm{RA}}$, as follows: ${ }^{4}$

$$
\eta_{\mathrm{RA}} \sim \frac{\beta}{C} \frac{\delta H}{\delta t}
$$

where $\beta$ is the isobaric volume expansion, $C$ is the specific heat coefficient, $H$ is the absorbed power density, which is proportional to the heating rate. The contrast could be improved not only due to the increase of absorption, but also due to the increase of $\beta$ and the decrease of $C$. This can be achieved by filling the pores with different liquids or polymers.

\section{Conclusions}

In conclusion, we showed that Si NPs can act as efficient contrast agents for RF-induced hyperthermia and TA imaging. Both the heating and the TA response of Si NP suspensions were proven to be mainly dependent on their conductivity, which can be tuned by ion concentration, controlled by the degree of Si NP dissolution. Thus the dissolution of Si NPs has an advantage over dissolution of other non-dissolvable solidstate NPs, such as gold, iron oxide, gadolinium and graphenebased NPs. Those effects were explained by the proposed model, which considers the observed temperature increase as induced by oscillations of solvated ions in the electric field of RF radiation. The model explains the linear relationship between the maximum heating rate and frequency of the $\mathrm{RF}$ radiation in the $\mathrm{MHz}$ frequency range. Taking into account the simulated heating rates for different biological tissues, we estimated the contrast between them and suspensions of Si NPs. According to the simulations, the most convenient operating frequency was about $70 \mathrm{MHz}$, because these conditions gave a contrast of $25 \%$ between Si NPs and a tissue and this value did not depend on the type of biological tissue. The obtained experimental and theoretical results can be useful for the design and development of new contrast agents for thermoacoustics and solid sensitizers for the RF hyperthermia of cancer.

\section{Acknowledgements}

M. Gongalsky acknowledges the Vladimir Vernadskij Program 2015 (\#57197893) supported by the German Academic Exchange Service (DAAD). The authors thank P. A. Forsh, A. S. Ilin and M. N. Martyshov for their help with impedance spectrometry measurements; A. V. Kabashin, Y. Ryabchikov and M. Sentis for the preparation of $\mathrm{Au}$ and ASi NP suspensions; V. P. Savinov for providing the RF generators of 1.2, 4.1 and $40.68 \mathrm{MHz}$. K. T. acknowledges program UMNIK (\#9826GU2/2015, code 0021161) for the support of the research. V. T. acknowledges the financial support of his work on nanoparticles characterization and RF heating experiments from the Russian Science Foundation (Grant No. 14-50-00029). Part of the results of this research at IBMI was supported by the funding from the Deutsche Forschungsgemeinschaft (DFG), Germany [Reinhard Koselleck award "High resolution near-field thermoacoustic sensing and imaging”; NT 3/9-1]. Y. H. thanks the CSC Fellowship (201306960006) for support.

\section{References}

1 T. V. Bartolotta, A. Taibbi, M. Midiri and M. De Maria, Abdom. imaging, 2008, 33, 501-511.

2 M. Omata, R. Tateishi, H. Yoshida and S. Shiina, Gastroenterology, 2004, 127, S159-S166.

3 G. Ku and L. V. Wang, Med. Phys., 2001, 28, 4.

4 D. Razansky, S. Kellnberger and V. Ntziachristos, Med. Phys., 2010, 37, 4602-4607.

5 D. E. Dupuy and S. N. Goldberg, J. Vasc. Interv. Radiol., 2001, 12, 1135-1148.

6 T. Boehm, A. Malich, S. N. Goldberg, J. R. Reichenbach, I. Hilger, P. Hauff, M. Reinhardt, M. Fleck and W. A. Kaiser, Radiology, 2002, 222, 805-813. 
7 M. Omar, S. Kellnberger, G. Sergiadis, D. Razansky and V. Ntziachristos, Med. Phys., 2012, 39, 4460-4466.

8 C. J. Gannon, C. R. Patra, R. Bhattacharya, P. Mukherjee and S. A. Curley, J. Nanobiotechnol., 2008, 6, 2.

9 D. E. Kruse, D. N. Stephens, H. A. Lindfors, E. S. Ingham, E. E. Paoli and K. W. Ferrara, IEEE Trans. Biomed. Eng., 2011, 58, 2002-2012.

10 E. S. Glazer, K. L. Massey, C. Zhu and S. A. Curley, Surgery, 2010, 148, 319-324.

11 J. Cardinal, J. R. Klune, E. Chory, G. Jeyabalan, J. S. Kanzius, M. Nalesnik and D. A. Geller, Surgery, 2008, 144, 125-132.

12 X. Liu, H.-j. Chen, X. Chen, C. Parini and D. Wen, Nanoscale, 2012, 4, 3945.

13 H. K. Kim, G. W. Hanson and D. A. Geller, Science, 2013, 340, 441-442.

14 C. H. Moran, S. M. Wainerdi, T. K. Cherukuri, C. Kittrell, B. J. Wiley, N. W. Nicholas, S. A. Curley, J. S. Kanzius and P. Cherukuri, Nano Res., 2009, 2, 400-405.

15 G. W. Hanson, R. C. Monreal and S. P. Apell, J. Appl. Phys., 2011, 109, 124306.

16 S. J. Corr, M. Raoof, Y. Mackeyev, S. Phounsavath, M. A. Cheney, B. T. Cisneros, M. Shur, M. Gozin, P. J. McNally, L. J. Wilson and S. A. Curley, J. Phys. Chem. C, 2012, 116, 24380-24389.

17 K. P. Tamarov, A. P. Kanavin, V. Y. Timoshenko, A. V. Kabashin and I. N. Zavestovskaya, Proc. SPIE, 2016, 973706.

18 K. P. Tamarov, L. A. Osminkina, S. V. Zinovyev, K. A. Maximova, J. V. Kargina, M. B. Gongalsky, Y. Ryabchikov, A. Al-Kattan, A. P. Sviridov, M. Sentis, A. V. Ivanov, V. N. Nikiforov, A. V. Kabashin and V. Y. Timoshenko, Sci. Rep., 2014, 4, 7034.

19 L. A. Osminkina, K. P. Tamarov, A. P. Sviridov, R. A. Galkin, M. B. Gongalsky, V. V. Solovyev, A. A. Kudryavtsev and V. Y. Timoshenko, J. Biophotonics, 2012, 5, 529-535.

20 L. Osminkina, K. Gonchar, V. Marshov, K. Bunkov, D. Petrov, L. Golovan, F. Talkenberg, V. Sivakov and V. Timoshenko, Nanoscale Res. Lett., 2012, 7, 1-6.

21 R. Herino, G. Bomchil, K. Barla, C. Bertrand and J. L. Ginoux, J. Electrochem. Soc., 1987, 134, 1994-2000.

22 L. A. Osminkina, V. A. Sivakov, G. A. Mysov, V. A. Georgobiani, U. Natashina, F. Talkenberg, V. V. Solovyev, A. A. Kudryavtsev and V. Y. Timoshenko, Nanoscale Res. Lett., 2014, 9, 463.

23 A. V. Kabashin and M. Meunier, J. Appl. Phys., 2003, 94, 7941.

24 P. Blandin, K. a. Maximova, M. B. Gongalsky, J. F. SanchezRoyo, V. S. Chirvony, M. Sentis, V. Y. Timoshenko and A. V. Kabashin, J. Mater. Chem. B, 2013, 1, 2489.

25 D. Rioux, M. Laferriè̀Ăre, A. Douplik, D. Shah, L. Lilge, A. V. Kabashin and M. M. Meunier, J. Biomed. Opt., 2009, 14, 021010.
26 V. Artemov, A. Volkov and N. Sysoev, EPL, 2015, 109, 26002. 27 N. C. Lara, A. A. Haider, L. J. Wilson, S. A. Curley and S. J. Corr, Appl. Phys. Lett., 2017, 110, 013701.

28 N. C. Lara, A. A. Haider, J. C. Ho, L. J. Wilson, A. R. Barron, S. A. Curley, S. J. Corr, A. R. Barron, L. J. Wilson, S. A. Curley, S. A. Curley, M. Raoof, D. T. Nguyen, Y. Zhang, L. J. Wilson, H. Summers, P. Rees, S. A. Curley and R. E. Serda, Chem. Commun., 2016, 52, 12630-12633.

29 R. Buchner, J. Barthel and J. Stauber, Chem. Phys. Lett., 1999, 306, 57-63.

30 P. A. Hasgall, E. Neufeld, M. Gosselin, A. Klingenböck and N. Kuster, IT IS Database for thermal and electromagnetic parameters of biological tissues, http://www.itis.ethz.ch/data base, 2013, version, 2, accessed, 2014.

31 M. J. Kogan, N. G. Bastus, R. Amigo, D. Grillo-Bosch, E. Araya, A. Turiel, A. Labarta, E. Giralt and V. F. Puntes, Nano Lett., 2006, 6, 110-115.

32 E. Sassaroli, K. C. P. Li and B. E. O’Neill, J. Phys. D: Appl. Phys., 2012, 45, 75303-75317.

33 G. Sonavane, K. Tomoda and K. Makino, Colloids Surf., B, 2008, 66, 274-280.

34 M. Kaasalainen, E. Mäkilä, J. Riikonen, M. Kovalainen, K. Järvinen, K.-H. Herzig, V.-P. Lehto and J. Salonen, Int. J. Pharm., 2012, 431, 230-236.

35 T. M. Squires and M. Z. Bazant, J. Fluid Mech., 2004, 509, 217-252.

36 D. Li, Y. S. Jung, S. Tan, H. K. Kim, E. Chory and D. A. Geller, J. Colloid Interface Sci., 2011, 358, 47-53.

37 O. Ogunlade and P. Beard, Med. Phys., 2015, 42, 170-181.

38 J.-P. Sylvestre, S. Poulin, A. V. Kabashin, E. Sacher, M. Meunier and J. H. T. Luong, J. Phys. Chem. B, 2004, 108, 16864-16869.

39 E. J. Anglin, L. Cheng, W. R. Freeman and M. J. Sailor, Adv. Drug Delivery Rev., 2008, 60, 1266-1277.

40 Z. Chen, Z.-M. Cui, C.-Y. Cao, W.-D. He, L. Jiang and W.-G. Song, Langmuir, 2012, 28, 13452-13458.

41 K. Tamarov, W. Xu, L. Osminkina, S. Zinovyev, P. Soininen, A. Kudryavtsev, M. Gongalsky, A. Gaydarova, A. Närvänen, V. Timoshenko and V.-P. Lehto, J. Controlled Release, 2016, 241, 220-228.

42 R. Valiullin, P. Kortunov, J. Karger and V. Timoshenko, J. Chem. Phys., 2004, 120, 11804-11814.

43 G. Lalwani, X. Cai, L. Nie, L. V. Wang and B. Sitharaman, Photoacoustics, 2013, 1, 62-67.

44 M. Pramanik, M. Swierczewska, D. Green, B. Sitharaman and L. V. Wang, J. Biomed. Opt., 2009, 14, 034018.

45 L. Nie, Z. Ou, S. Yang and D. Xing, Med. Phys., 2010, 37, 4193-4200.

46 H. Qin, S. Yang and D. Xing, Appl. Phys. Lett., 2012, 100, 033701. 